\title{
Sign Wave Analysis in Matrix Eigenvalue Problems
}

\author{
By K. M. Brown and P. Henrici
}

1. Introduction. In this paper the word "matrix" denotes a real (but not necessarily symmetric) matrix of order $N$; by "vector" we mean a column vector with real or complex elements. For any matrix $A$, the roots of the equation $\operatorname{det}(A-\lambda I)=0$ ( $I=$ unit matrix) are called the eigenvalues of $A$. By the multiplicity of an eigenvalue we mean its multiplicity as a root of the above polynomial equation. If $\lambda$ is an eigenvalue of $A$, then any nontrivial solution $x$ of the equation $(A-\lambda I) x=0$ is called an eigenvector (of $A$ ) associated with $\lambda$. An eigenvalue is a dominant eigenvalue of the matrix $A$ if its modulus is exceeded by the modulus of no other eigenvalue of $A$.

The power method ([1]; [3], p. 296; [5]; [7]; [9]; [10]) is generally recognized as a numerically efficient algorithm for determining the dominant eigenvalue(s) and associated eigenvector(s) of a matrix. We review the method briefly for the case where the matrix $A$ has a single dominant eigenvalue $\lambda$ with associated eigenvector $u$. (It is assumed that $\lambda$ has multiplicity one.) Denoting by the superscript ${ }^{T}$ the transpose of a vector or matrix, we let $v$ be an eigenvector of $A^{r}$ associated with the eigenvalue $\lambda$. Starting with any vector $x^{(0)}$ satisfying $v^{T} x^{(0)} \neq 0$, we now form by successive matrix-vector multiplications the vectors

$$
x^{(n+1)}=A x^{(n)}, \quad n=0,1,2, \cdots .
$$

Then, denoting by $a_{\nu}(\nu=1,2, \cdots, N)$ the components of a vector $a$, we have for every $\nu$ such that $u_{\nu} \neq 0$

$$
\lim _{n \rightarrow \infty} \frac{x_{\nu}^{(n+1)}}{x_{\nu}^{(n)}}=\lambda
$$

and furthermore, as $n \rightarrow \infty$,

$$
{x_{1}}^{(n)}: x_{2}^{(n)}: \cdots: x_{N}^{(n)} \rightarrow u_{1}: u_{2}: \cdots u_{N}^{*}
$$

The convergence of the process can be sped up by devices such as shift of the origin [10], fractional iteration [8], and the $\delta^{2}$-process [1]. Statements similar to the above still hold if the multiplicity of $\lambda$ is greater than one, but the convergence may then be slow due to the presence of nonlinear divisors. Once $\lambda, u$, and the associated eigenvector of $A^{T}$ have been determined, one can, by a process known as deflation, construct a matrix $A_{1}$ whose eigenvalues and eigenvectors are the same as those of $A$, except that the eigenvalue $\lambda$ is replaced by 0 . The above process can

Received December 17, 1961. The preparation of this paper was sponsored by the U. S. Army Research Office at Durham, N. C.

* This notation means that

$$
\frac{x_{\nu}^{(n)}}{\left[\sum_{\mu=1}^{N}\left|x_{\mu}^{(n)}\right|^{2}\right]^{1 / 2}} \rightarrow \frac{u_{\nu}}{\left[\sum_{\mu=1}^{N}\left|u_{\mu}\right|^{2}\right]^{1 / 2}} \quad(n \rightarrow \infty, \nu=1,2, \cdots, N)
$$


then be repeated; if the matrices $A_{1}, A_{2}, \cdots$ all have single dominant eigenvalues, the above method yields successively all eigenvalues and eigenvectors of the matrix $A$.

2. Conjugate Complex Dominant Eigenvalues. In the present note we wish to deal with the case where the matrix $A$ has exactly two dominant eigenvalues, both simple, represented by the pair of conjugate complex numbers $\lambda=\rho e^{i \varphi}$ and $\bar{\lambda}=\rho e^{-i \varphi}$, where $\rho>0,0<\varphi<\pi$. The eigenvectors associated with $\lambda$ and with $\bar{\lambda}$ may then be assumed to be conjugate complex vectors also. We shall denote them by $u$ and $\bar{u}$, where the components $u_{\nu}$ of $u$ are given by

$$
u_{\nu}=r_{\nu} e^{i \varphi_{\nu}}, \quad \quad \nu=1,2, \cdots, N .
$$

In view of the fact that the eigenvectors are determined only up to a non-zero factor (which in the present case may even be complex), it should be noted that the $r_{\nu}$ are determined only up to a positive factor, and the $\varphi_{\nu}$ only up to a common additive constant modulo $2 \pi$. Indicating by the superscript ${ }^{H}$ the conjugate transpose of a complex vector or matrix, we denote by $v$ the eigenvector of $A^{H}$ belonging to $\lambda$, normalized such that $v^{H} u=1$.

One of the methods for determining conjugate complex eigenvalues and corresponding eigenvectors from the sequence $\left\{x^{(n)}\right\}$ that have been proposed ([3], p. 296 ; [9]) is known to be numerically unstable for small values of $\varphi$ [9]. In Section 3 below we propose an alternate method that appears to be uniformly accurate for all values of $\varphi$. In addition, the method yields very good approximations for both $\varphi$ and the $\varphi_{\nu}$ almost without computation, by mere inspection of the signs of the sequences of the components of the (real) vectors $x^{(n)}$.

3. Sign Waves. It is known ([2], p. 28j) that the presence of a pair of conjugate complex dominant eigenvalues is indicated by the occurrence of sign changes in the sequences $\left\{x_{\nu}{ }^{(n)}\right\}$. For a certain matrix of order 6 , the signs of the $x_{\nu}{ }^{(n)}$ were distributed as follows:

\begin{tabular}{c|cccccc}
$\nu$ & 1 & 2 & 3 & 4 & 5 & 6 \\
\hline$n=0$ & + & + & + & + & + & + \\
1 & + & + & + & - & + & + \\
2 & - & - & - & - & - & + \\
3 & + & - & - & + & - & - \\
4 & + & + & + & - & + & + \\
5 & - & - & + & - & - & + \\
6 & - & - & - & + & - & - \\
7 & - & - & - & + & - & - \\
8 & + & + & + & - & + & + \\
9 & + & + & + & - & + & + \\
10 & & & & & &
\end{tabular}

Our method consists in exploring systematically the period and relative location of these sign waves. We ascribe a sign to all elements of the $N$ sequences 
$\left\{x_{\nu}{ }^{(n)}\right\}(\nu=1, \cdots, N)$. A zero element is assigned the sign of the first nonzero element following it. (If there is no such element, the sign is irrelevant for the following theory.) For $k=1,2, \cdots$ we denote by $n_{\nu}{ }^{(k)}$ the index of the element in the sequence $\left\{x_{\nu}{ }^{(n)}\right\}$ at which the sign changes from minus to plus for the $k$ th time, i.e., at which

$$
\operatorname{sign} x_{\nu}^{(n-1)}=-1, \quad \operatorname{sign} x_{\nu}^{(n)}=+1 .
$$

(The indices $n_{\nu}{ }^{(k)}$ mark the beginnings of the $k$ th sign wave in the sequence $\left\{x_{\nu}{ }^{(n)}\right\}$. In the example given above, $n_{1}^{(1)}=4, n_{1}^{(2)}=9, n_{5}^{(2)}=9$.) For $\nu, \mu=1,2, \cdots$, $N$ and $k=1,2, \cdots$ we put

$$
\begin{aligned}
P_{\nu}{ }^{(k)} & =n_{\nu}{ }^{(k+1)}-n_{\nu}{ }^{(k)}, \\
\delta_{\nu \mu}{ }^{(k)} & =n_{\mu}{ }^{(k)}-n_{\mu}{ }^{(k)} .
\end{aligned}
$$

$\left(P_{\nu}{ }^{(k)}\right.$ indicates the length of the $k$ th sign wave in the $\nu$ th component, and $\delta_{\nu \mu}^{(k)}$ represents the phase difference between the $k$ th sign waves in the $\nu$ th and $\mu$ th components. In the above example, $P_{3}^{(1)}=4, \delta_{42}^{(2)}=-2$.) We finally require the quantities

$$
\Delta_{\nu}^{(n)}=\left[x_{\nu}^{(n)}\right]^{2}-x_{\nu}^{(n+1)} x_{\nu}^{(n-1)} .
$$

With these definitions, we can state the following result:

THEOREM. Let the matrix A satisfy the conditions stated at the beginning of Section 2, and let the vector $x^{(0)}$ be such that $v^{H} x^{(0)} \neq 0$. Then the vectors $x^{(n)}$ defined by (1) satisfy

$$
\Delta_{1}^{(n)}: \Delta_{2}{ }^{(n)}: \cdots: \Delta_{N}{ }^{(n)} \rightarrow r_{1}{ }^{2}: r_{2}{ }^{2}: \cdots: r_{N}{ }^{2} .
$$

For every $\nu$ such that $r_{\nu} \neq 0$, the following two statements hold:

$$
\begin{gathered}
\rho^{2}=\lim _{n \rightarrow \infty} \frac{\Delta_{\nu}{ }^{(n+1)}}{\Delta_{\nu}{ }^{(n)}} ; \\
\lim _{k \rightarrow \infty} \frac{P_{\nu}^{(1)}+P_{\nu}^{(2)}+\cdots+P_{\nu}^{(k)}}{k}=P
\end{gathered}
$$

exists, and

$$
\varphi=\frac{2 \pi}{P}
$$

For all $\nu$ and $\mu$ such that $r_{\nu} \neq 0, r_{\mu} \neq 0$, the following two statements hold:

(iv) If $\varphi / 2 \pi$ is irrational, then

$$
\lim _{k \rightarrow \infty} \frac{\delta_{\nu \mu}^{(1)}+\delta_{\nu \mu}^{(2)}+\cdots+\delta_{\nu \mu}^{(k)}}{k P} \equiv \frac{\varphi_{\mu}-\varphi_{\nu}}{2 \pi}(\bmod 1)
$$

(v) If $\varphi / 2 \pi=p / q$ is rational $((p, q)=1)$ it can only be asserted that, for some integer $l$, both the limit superior and the limit inferior of

$$
\frac{\delta_{\nu \mu}^{(1)}+\delta_{\nu \mu}^{(2)}+\cdots+\delta_{\nu \mu}^{(k)}}{k P}
$$

as $k \rightarrow \infty$ differ by at most $1 / q$ from

$$
\frac{\varphi_{\mu}-\varphi_{\nu}}{2 \pi}+l .
$$


4. Proof of the Theorem. Under the hypotheses of the theorem the matrix $A$ can be represented in the form

$$
A=\lambda u v^{T}+\bar{\lambda} \bar{u} v^{H}+A_{1}
$$

where $A_{1}$ is a matrix whose eigenvalues lie inside a circle of radius $q \rho, 0<q<1$. If $v^{T} x^{(0)}=a e^{i \alpha}$, where $a>0$, we have

$$
x^{(n)}=a\left(\lambda^{n} u e^{i \alpha}+\bar{\lambda}^{n} \bar{u} e^{-i a}+w^{(n)}\right)
$$

where the components of $w^{(n)}$ are bounded by $C q^{n} \rho^{n}$ with a suitable constant $C$. Hence

$$
x_{\nu}{ }^{(n)}=2 a \rho^{n}\left\{r_{\nu} \cos \left(n \varphi+\varphi_{\nu}+\alpha\right)+\epsilon_{\nu}{ }^{(n)}\right\}
$$

where

$$
\left|\epsilon_{\nu}{ }^{(n)}\right| \leqq C q^{n}
$$

A simple calculation now yields

$$
\Delta_{\nu}^{(n)}=\frac{4 a^{2} \rho^{2 n}}{(\sin \varphi)^{2}}\left(r_{\nu}{ }^{2}+\eta_{\nu}{ }^{(n)}\right)
$$

where

$$
\left|\eta_{\nu}^{(n)}\right| \leqq 2 C q^{n} \sin ^{2} \varphi\left(2 r_{\nu}+C q^{n}\right)
$$

and hence $\eta_{\nu}{ }^{(n)} \rightarrow 0$ as $n \rightarrow \infty$. The relations (i) and, if $r_{\nu} \neq 0$, (ii) now follow immediately.

For the proof of the remaining statements of the theorem a modified version of (1) is required. Assume the integer $n^{\prime}$ is such that, for all $\nu$ satisfying $r_{\nu} \neq 0$,

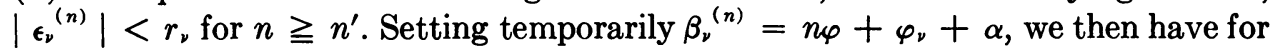
$n \geqq n^{\prime}$

$$
\begin{aligned}
\left(2 a \rho^{n}\right)^{-1} x_{\nu}{ }^{(n)} & =\operatorname{Re}\left\{r_{\nu} e^{i \beta_{\nu}(n)}+\epsilon_{\nu}{ }^{(n)}\right\} \\
& =\operatorname{Re}\left\{e^{i \beta_{\nu}(n)}\left[r_{\nu}+e^{-i \beta_{\nu}(n)} \epsilon_{\nu}{ }^{(n)}\right]\right\} \\
& =\left|r_{\nu}+e^{-i \beta_{\nu}(n)} \epsilon_{\nu}{ }^{(n)}\right| \operatorname{Re}\left\{e^{i\left(\beta_{\nu}(n)-\theta_{\nu}(n)\right)}\right\}
\end{aligned}
$$

where

$$
\tan \theta_{\nu}{ }^{(n)}=\frac{\epsilon_{\nu}{ }^{(n)} \sin \beta_{\nu}{ }^{(n)}}{r_{\nu}+\epsilon_{\nu}{ }^{(n)} \cos \beta_{\nu}{ }^{(n)}}, \quad\left|\theta_{\nu}{ }^{(n)}\right|<\frac{\pi}{2} .
$$

Hence

$$
x_{\nu}{ }^{(n)}=A_{\nu}{ }^{(n)} \sin \phi_{\nu}{ }^{(n)}
$$

where

$$
\begin{aligned}
& A_{\nu}{ }^{(n)}=2 a \rho^{n}\left|r_{\nu}+e^{-i \beta_{\nu}{ }^{(n)}} \epsilon_{\nu}{ }^{(n)}\right|>0, \\
& \phi_{\nu}{ }^{(n)}=n \varphi+\varphi_{\nu}+\alpha+\frac{\pi}{2}-\theta_{\nu}{ }^{(n)} .
\end{aligned}
$$

Formula (4) serves to determine the sign of $x_{\nu}{ }^{(n)}$ as a function of $n$. 
We shall require an explicit formula for $n_{\nu}{ }^{(k)}$ valid for large $k$. It follows from (2) and (3) that $\theta_{\nu}^{(n)} \rightarrow 0$ for $n \rightarrow \infty$. Let $n^{\prime \prime}$ be such that

$$
\left|\theta_{\nu}^{(n)}\right| \leqq \frac{1}{2} \operatorname{Min}(\varphi, \pi-\varphi), \quad n>n^{\prime \prime}
$$

for all $\nu$ satisfying $r_{\nu} \neq 0$. We then have

$$
0<\phi_{\nu}{ }^{(n+1)}-\phi_{\nu}{ }^{(n)}<\pi, \quad n>n^{\prime \prime},
$$

i.e., the sequence $\left\{\phi_{\nu}{ }^{(n)}\right\}$ is monotonically increasing and assumes a value in every (open) interval of length $\pi$. Let $k_{\nu}$ denote the smallest integer such that

$$
n_{\nu}{ }^{\left(k_{\nu}\right)}>n^{\prime \prime} \text {. }
$$

By (5) and by the definition of $n_{\nu}{ }^{(k)}$, there exists an integer $m_{\nu}$ such that

$$
\begin{aligned}
\phi^{\left(n\left(k_{\nu}\right)\right)} & =n_{\nu}{ }^{\left(k_{\nu}\right)} \varphi+\varphi_{\nu}+\alpha+\frac{\pi}{2}-\theta_{\nu}{ }^{(n)} \geqq 2 m_{\nu} \pi, \\
\phi_{\nu}{ }^{\left(n\left(k_{\nu}\right)-1\right)} & =\left(n_{\nu}{ }^{\left(k_{\nu}\right)}-1\right) \varphi+\varphi_{\nu}+\alpha+\frac{\pi}{2}-\theta_{\nu}{ }^{(n-1)}<2 m_{\iota} \pi .
\end{aligned}
$$

More generally, for $m=0,1,2, \cdots$ we have

$$
\begin{gathered}
n_{\nu}{ }^{\left(k_{\nu}+m\right)} \varphi+\varphi_{\nu}+\alpha+\frac{\pi}{2}-\theta_{\nu}{ }^{(n)} \geqq 2\left(m_{\nu}+m\right) \pi \\
\left(n_{\nu}{ }^{\left(k_{\nu}+m\right)}-1\right) \varphi+\varphi_{\nu}+\alpha+\frac{\pi}{2}-\theta_{\nu}{ }^{(n-1)}<2\left(m_{\nu}+m\right) \pi .
\end{gathered}
$$

We denote, for any real number $a$, by $[a]$ the largest integer not exceeding $a$. We also set

$$
\psi_{\nu}=\varphi_{\nu}+\alpha+\frac{\pi}{2}+2 \pi\left(k_{\nu}-m_{\nu}\right)
$$

If $k=k_{\nu}+m \geqq k_{\nu}$, it then follows that

$$
n_{\nu}{ }^{(k)}=\left[\frac{2 \pi k-\psi_{\nu}+\theta_{\nu}^{(n)}}{\varphi}\right] \text {. }
$$

For the proof of (iii) we observe that

$$
n_{\nu}{ }^{(k)}=\frac{2 \pi k}{\varphi}+C_{\nu}{ }^{(k)}
$$

where the moduli of the numbers $C_{\nu}{ }^{(k)}$ are bounded. We have

$$
P_{\nu}{ }^{(1)}+P_{\nu}{ }^{(2)}+\cdots+P_{\nu}{ }^{(k)}=n_{\nu}{ }^{(k+1)}-n_{\nu}{ }^{(1)}
$$

and hence, using ( 7$)$, if $k \geqq k_{\nu}$,

$$
\frac{P_{\nu}{ }^{(1)}+P_{\nu}{ }^{(2)}+\cdots+P_{\nu}{ }^{(k)}}{k}=\frac{2 \pi}{\varphi}+\left(\frac{2 \pi}{\varphi}+C_{\nu}{ }^{(k)}-n_{\nu}{ }^{(1)}\right) \frac{1}{k} .
$$

The second term on the right tends to zero as $k \rightarrow \infty$, and (iii) follows. 
For the proof of (iv) we set

$$
N_{\nu}{ }^{(k)}=\frac{1}{2} k-\frac{\varphi}{2 \pi k}\left(n_{\nu}{ }^{(1)}+n_{\nu}{ }^{(2)}+\cdots+n_{\nu}{ }^{(k)}\right) .
$$

The following lemma is required:

Lемма. If $\varphi / 2 \pi$ is irrational, then there exists a constant $c$ such that for all $\nu$ satisfying $r_{\nu} \neq 0$

$$
\lim _{k \rightarrow \infty} N_{\nu}{ }^{(k)} \equiv \frac{\varphi_{\nu}}{2 \pi}+c(\bmod 1)
$$

The proof consists in showing that for a suitable integer $l_{\nu}$ and for every $\delta>0$

$$
\limsup _{k \rightarrow \infty} N_{\nu}^{(k)} \leqq \frac{\varphi_{\nu}}{2 \pi}+c+l_{\nu}+\delta
$$

and

$$
\liminf _{k \rightarrow \infty} N_{\nu}^{(k)} \geqq \frac{\varphi_{\nu}}{2 \pi}+c+l_{\nu}-\delta .
$$

For $k \geqq k_{\nu}$, let

where

$$
n_{\nu}{ }^{(k)}=p_{\nu}{ }^{(k)}+q_{\nu}{ }^{(k)}+s_{\nu}{ }^{(k)},
$$

$$
\begin{aligned}
p_{\nu}{ }^{(k)} & =\frac{2 \pi k-\psi_{\nu}}{\varphi}, \\
q_{\nu}{ }^{\left({ }^{2}\right)} & =\left[\frac{2 \pi k-\psi_{\nu}}{\varphi}\right]-\frac{2 \pi k-\varphi_{\nu}}{\varphi}, \\
s_{\nu}{ }^{(k)} & =\left[\frac{2 \pi k-\psi_{\nu}+\theta_{\nu}{ }^{(n)}}{\varphi}\right]-\left[\frac{2 \pi k-\psi_{\nu}}{\varphi}\right] .
\end{aligned}
$$

Let $\delta>0$ be given, and let $h$ be an integer such that

$$
\frac{\left|\theta^{(n)}\right|}{\varphi}<\delta \text { for } k \geqq h
$$

where $n=n_{\nu}{ }^{(k)}$. We then have

where

$$
N_{\nu}{ }^{(k)}=\frac{1}{2} k-\frac{1}{2 \pi k}\left\{M+\varphi \sum_{m=h}^{k}\left(p_{\nu}{ }^{(m)}+q_{\nu}{ }^{(m)}+s_{\nu}{ }^{(m)}\right)\right\},
$$

$$
M=\left(n_{\nu}{ }^{(1)}+n_{\nu}{ }^{(2)}+\cdots+n_{\nu}{ }^{(h-1)}\right) \varphi .
$$

Since $M$ does not depend on $k, k^{-1} M \rightarrow 0$ as $k \rightarrow \infty$. An easy computation yields

$$
\begin{aligned}
\frac{1}{2} k-\frac{\varphi}{2 \pi k}\left(p_{\nu}{ }^{(h)}+p_{\nu}{ }^{(h+1)}+\cdots+\right. & \left.p_{\nu}{ }^{(k)}\right) \\
& =-\frac{1}{2}+\frac{\psi_{\nu}}{2 \pi}+\frac{1}{2 \pi k}\left\{h(h-1)-(h-1) \psi_{\nu}\right\} .
\end{aligned}
$$

The limit of this expression as $k \rightarrow \infty$ exists and equals $-\frac{1}{2}+\psi_{\nu} / 2 \pi$.

Since $\pi / \varphi$ is irrational, the numbers ${q_{\nu}}^{(k)}$ are equidistributed in the interval $(-1,0]$ according to a classical theorem by $\mathrm{H}$. Weyl (see [6], p. 71, 234). Hence

$$
\lim _{k \rightarrow \infty} \frac{1}{k}\left(q_{\nu}{ }^{(h)}+q_{\nu}{ }^{(h+1)}+\cdots+q_{\nu}{ }^{(k)}\right)=-\frac{1}{2} .
$$


According to the definition of $h$, the numbers $s_{\nu}{ }^{(k)}$ can differ from 0 only if $q_{\nu}{ }^{(k)}$ lies either in the interval $(-1,-1+\delta)$ or in $(-\delta, 0]$. In either case, $\left|s_{\nu}{ }^{(k)}\right| \leqq 1$. According to Weyl's theorem, the number of times either possibility occurs is asymptotic to $k \delta$ as $k \rightarrow \infty$. It follows that

$$
\limsup _{k \rightarrow \infty} \frac{1}{k}\left|s_{\nu}^{\left({ }^{(h)}\right.}+s_{\nu}{ }^{(h+1)}+\cdots+s_{\nu}{ }^{(k)}\right| \leqq \delta .
$$

Gathering the above results, we find that

$$
\begin{gathered}
\limsup _{k \rightarrow \infty} N_{\nu}{ }^{(k)} \leqq \frac{\psi_{\nu}}{2 \pi}-\frac{1}{2}+\frac{\varphi}{2 \pi}\left(-\frac{1}{2}+\delta\right), \\
\liminf _{k \rightarrow \infty} N_{\nu}{ }^{(k)} \geqq \frac{\psi_{\nu}}{2 \pi}-\frac{1}{2}+\frac{\varphi}{2 \pi}\left(-\frac{1}{2}-\delta\right) .
\end{gathered}
$$

In view of $(6)$, this establishes the relations (8) with

$$
c=\frac{\alpha}{2 \pi}-\frac{\varphi}{4 \pi}-\frac{1}{4}, \quad l_{\nu}=k_{v}-m_{\nu} .
$$

The statement of the lemma now follows in view of the fact that the above is true for arbitrary $\delta>0$.

Statement (iv) of the theorem now follows by observing the relation

$$
\frac{\delta_{\nu \mu}^{(1)}+\delta_{\nu \mu}^{(2)}+\cdots+\delta_{\nu \mu}^{(k)}}{k P}=N_{\mu}{ }^{(k)}-N_{\nu}{ }^{(k)}
$$

and letting $k \rightarrow \infty$.

If $2 \pi / \varphi=q / p$ is rational and if $(p, q)=1$, then the numbers $q_{\nu}{ }^{(k)}$ are no longer equidistributed in $(-1,0]$, but take on with equal frequency ([4], p. 51) the $p$ distinct values

$$
-\frac{m}{p}-\xi, \quad m=0,1, \cdots, p-1,
$$

where $\xi$ is some number depending on $\varphi_{\nu}, 0 \leqq \xi<1 / p$. It follows that

$$
\lim _{k \rightarrow \infty} \frac{1}{k}\left(q_{\nu}{ }^{(h)}+q_{\nu}{ }^{(h+1)}+\cdots+q_{\nu}{ }^{(k)}\right)=\frac{1-p}{2 p}-\xi .
$$

If $\xi \neq 0$ in $(10)$, the numbers $s_{\nu}{ }^{(k)}$ are all zero for $k$ sufficiently large, and thus

$$
\lim _{k \rightarrow \infty} \frac{1}{k}\left(s_{\nu}{ }^{(h)}+s_{\nu}{ }^{(h+1)}+\cdots+s_{\nu}{ }^{(k)}\right)=0 .
$$

If $\xi=0$, then $s_{\nu}{ }^{(k)}=-1$ if $q_{\nu}{ }^{(k)}=0$ and $\theta_{\nu}{ }^{(n)}<0$, and $s_{\nu}{ }^{(k)}=0$ otherwise. We have $q_{\nu}{ }^{(k)}=0$ every $p$ th time for $k$ large, thus

$$
\begin{aligned}
& \limsup _{k \rightarrow \infty} \frac{1}{k}\left(s_{\nu}{ }^{(h)}+s_{\nu}{ }^{(h+1)}+\cdots+s_{\nu}{ }^{(k)}\right) \leqq 0, \\
& \liminf _{k \rightarrow \infty} \frac{1}{k}\left(s_{\nu}{ }^{(h)}+s_{\nu}{ }^{(h+1)}+\cdots+s_{\nu}{ }^{(k)}\right) \geqq-\frac{1}{p} .
\end{aligned}
$$


Thus in any case, if $2 \pi / \varphi$ is rational,

$$
\begin{aligned}
& \lim _{k \rightarrow \infty} \sup _{\nu}{ }_{\nu}^{(k)} \leqq \frac{\psi_{\nu}}{2 \pi}-\frac{1}{2}-\frac{\varphi}{4 \pi}\left(1-\frac{1}{p}\right), \\
& \liminf _{k \rightarrow \infty} N_{\nu}{ }^{(k)} \geqq \frac{\psi_{\nu}}{2 \pi}-\frac{1}{2}-\frac{\varphi}{4 \pi}\left(1+\frac{1}{p}\right) .
\end{aligned}
$$

From these relations statement ( $v$ ) of the theorem follows as above by observing (9) and using the relations

$$
\begin{aligned}
& \limsup _{k \rightarrow \infty} \frac{\delta_{\nu \mu}^{(1)}+\delta_{\nu \mu}^{(2)}+\cdots+\delta_{\nu \mu}^{(k)}}{k P} \\
& \leqq \limsup _{k \rightarrow \infty} N_{\mu}^{(k)}-\liminf _{k \rightarrow \infty} N_{\nu}{ }^{(k)} \\
& \leqq \frac{\psi_{\mu}-\psi_{\nu}}{2 \pi}+\frac{\varphi}{2 \pi p} \equiv \frac{\varphi_{\mu}-\varphi_{\nu}}{2 \pi}+\frac{1}{q}(\bmod 1)
\end{aligned}
$$

and a similar relation for the limit inferior.

5. Numerical Results.

1) As a basis for the numerical experiments we used the following $6 \times 6$ matrix $A$ depending on two real parameters $a$ and $b$, not both 0 :

$$
\begin{aligned}
& A=\alpha U D U^{H}, \quad \text { where } \\
& \alpha=\frac{1}{|a|+|b|} \\
& U=\left(\begin{array}{cccccc}
\frac{1}{\sqrt{6}} & \frac{1}{\sqrt{6}} & \frac{1}{\sqrt{6}} & \frac{1}{\sqrt{6}} & \frac{1}{\sqrt{6}} & \frac{1}{\sqrt{6}} \\
\frac{1}{\sqrt{6}} & \frac{1}{\sqrt{6}} & \frac{-1}{\sqrt{6}} & \frac{-1}{\sqrt{6}} & \frac{1}{\sqrt{6}} & \frac{-1}{\sqrt{6}} \\
\frac{2}{\sqrt{12}} & \frac{-1}{\sqrt{12}} & \frac{2}{\sqrt{12}} & \frac{-1}{\sqrt{12}} & \frac{-1}{\sqrt{12}} & \frac{-1}{\sqrt{12}} \\
\frac{-1}{\sqrt{12}} & \frac{2}{\sqrt{12}} & \frac{1}{\sqrt{12}} & \frac{1}{\sqrt{12}} & \frac{-1}{\sqrt{12}} & \frac{-2}{\sqrt{12}} \\
\frac{1}{\sqrt{8}} & \frac{1}{\sqrt{8}} & \frac{-1}{\sqrt{8}} & 0 & \frac{-2}{\sqrt{8}} & \frac{1}{\sqrt{8}} \\
\frac{1}{\sqrt{8}} & \frac{-1}{\sqrt{8}} & \frac{-1}{\sqrt{8}} & \frac{2}{\sqrt{8}} & 0 & \frac{-1}{\sqrt{8}}
\end{array}\right)
\end{aligned}
$$

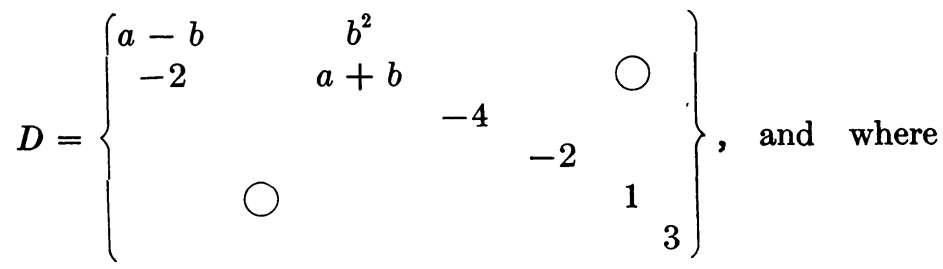

$U^{H}$ is the conjugate transpose of $U$. 
The eigenvalues of $A$ are seen to be:

$$
\alpha(a+b i), \quad \alpha(a-b i), \quad-4 \alpha, \quad-2 \alpha, \quad \alpha, \quad \text { and } 3 \alpha .
$$

2) For $a=6, b=\frac{1}{2}$, the resulting matrix $A$ was as follows:

$$
\left[\begin{array}{clrrcc}
0.21 i 5385 & 0.3653846 & -0.1767767 & -0.05439283 & 0.3386381 & -0.1387861 \\
0.3653846 & 0.2115385 & 0.1495803 & 0.3807498 & 0.02775722 & -0.005551444 \\
-0.05439283 & 0.2719641 & 0.2307692 & -0.3076923 & 0.2198260 & 0.5338631 \\
-0.1767767 & 0.2583659 & -0.3942308 & 0.5384615 & 0.05103104 & -0.2551552 \\
0.3386381 & 0.02775722 & 0.1138384 & 0.1570186 & 0.2548077 & -0.1971154 \\
-0.03886011 & 0.09437457 & 0.5691923 & -0.2198260 & -0.1105769 & 0.09134615
\end{array}\right]
$$

For this matrix, the following results were obtained:

Eigenvalue

Computed Absolute Value Computed Argument 0.926276

0.083221 radians

Actual

Absolute Value

Actual Argument

0.926276

0.083140 radians

\section{Eigenvector*}

Computed Absolute Value
1
1.00000
0.392232
0.980581
0.866025
0.537086

Computed

Argument

$$
33.45^{\circ}
$$

$33.45^{\circ}$

$-90.00^{\circ}$

$52.53^{\circ}$

$33.45^{\circ}$

$-115.84^{\circ}$
Actual Absolute Value

\section{1 \\ 1.00000}

0.392232

0.980580

0.866025

0.537086
Actual

Argument

$33.68^{\circ}$

$33.68^{\circ}$

$-90.00^{\circ}$

$53.12^{\circ}$

$33.68^{\circ}$

$-116.57^{\circ}$

3) Other cases:

a) For $a=\frac{1}{2}$ and $b=5$, the results were as follows:

Eigenvalue

$\begin{array}{cccc}\begin{array}{c}\text { Computed } \\ \text { Absolute Value }\end{array} & \begin{array}{c}\text { Computed } \\ \text { Argument }\end{array} & \begin{array}{c}\text { Actual Absolute } \\ \text { Value }\end{array} & \begin{array}{c}\text { Actual } \\ \text { Argument }\end{array} \\ 0.913625 & 1.46980 \text { radians } & 0.913625 & 1.47113 \text { radians }\end{array}$

Eigenvector

$\begin{array}{cccc}\begin{array}{c}\text { Computed Absolute } \\ \text { Value }\end{array} & \begin{array}{c}\text { Computed } \\ \text { Argument }\end{array} & \begin{array}{c}\text { Actual Absolute } \\ \text { Value }\end{array} & \begin{array}{c}\text { Actual } \\ \text { Argument }\end{array} \\ 1 & 0 \text { radians } & 1 & 0 \text { radians } \\ 1.00000 & 0.0000 & 1.00000 & 0.0000 \\ 1.05267 & -0.2815 & 1.05267 & -.2750 \\ 0.419137 & 2.377 & 0.419137 & 2.324 \\ 0.866025 & 0.0000 & 0.866025 & 0.0000 \\ 0.587022 & -0.4378 & 0.587022 & -0.4101\end{array}$

b) The case $a=4, b=\frac{1}{2}$ proved to be of interest. Letting $\lambda_{1}, \cdots, \lambda_{6}$ denote the actual (theoretical) eigenvalues of the matrix corresponding to this case, it turns out that $\left|\lambda_{1}\right|=\left|\lambda_{2}\right|=0.8958064$ and $\left|\lambda_{3}\right|=0.8888888$, so that $\lambda_{3}$ is close

* Arguments in 2 were normalized so that the argument of the third componert was $-90^{\circ}$. 
to $\lambda_{1}$ both in location and absolute value. The numerical process for finding the absolute value of $\lambda_{1}$ did not converge in this case, but the numerical procedure for finding the argument of $\lambda_{1}$ yielded 0.12433 radians as compared with the actual value of 0.12436 radians; i.e., the angle was obtained as accurately in this case as in cases where $\left|\lambda_{1}\right|=\left|\lambda_{2}\right| \gg\left|\lambda_{3}\right|>\cdots$.

c) The case $a=0, b=5$ yielded the eigenvalue (i.e., absolute value and argument) exactly.

Note: In each numerical example considered, $(1,1,1,1,1,1)$ was used as the starting vector, and $\varphi$ was determined from the average period of all components. The latter procedure was found to yield the angle $\varphi$ more accurately than when the average period of just one component was used.

Space Technology Laboratories, Inc.

Los Angeles, California

University of California

Los Angeles, California

1. A. C. Aitren, "Studies in practical mathematics, II. The evaluation of the latent roots and latent vectors of a matrix," Proc. Roy. Soc. Edinburgh, v. 57, 1937, p. 269-304.

2. E. Bodewig, Matrix Calculus, North Holland Publishing Co., Amsterdam, 1959.

3. L. Collat Tz, Eigenwertaufgaben mit Technischen Anwendungen, Leipzig, 1949.

4. G. H. HARD Y E. M. WRIGHT, An Introduction to the Theory of Numbers, third edition, Oxford, 1954 .

5. E. E. Osborne, "On acceleration and matrix deflation processes used with the power method," J. Soc. Indust. Appl. Math., v. 6, 1958, p. 279-287.

6. G. Polya \& G. Szegö, Aufgaben und Lehrsätze der Analysis, v. I., Springer, Berlin, 1954.

7. R. von Mises \& H. Geiringer, "Praktische Verfahren der Gleichungsauflösung," Z. Angew. Math. Mech., v. 9, 1929, p. 58-77, p. 152-164.

8. H. WiELANDT, "Das Iterationsverfahren bei nicht selbstadjungierten linearen Eigenwertaufgaben," Math. Z., v. 50, 1954, p. 93-143.

9. J. H. Wilkinson," "The calculation of the latent roots and vectors of matrices on the Pilot model of the ACE," Proc. Cambridge Philos. Soc., v. 50, 1954, p. 536-566.

10. J. H. WiLkINSON, "The use of iterative methods for finding the latent roots and vectors of matrices," MTAC, v. 9, 1955, p. 184-191. 